TRANSACTIONS OF THE

AMERICAN MATHEMATICAL SOCIETY

Volume 360, Number 6, June 2008, Pages 3307-3326

S 0002-9947(08)04435-8

Article electronically published on January 30, 2008

\title{
FRAME REPRESENTATIONS AND PARSEVAL DUALS WITH APPLICATIONS TO GABOR FRAMES
}

\author{
DEGUANG HAN
}

\begin{abstract}
Let $\left\{x_{n}\right\}$ be a frame for a Hilbert space $H$. We investigate the conditions under which there exists a dual frame for $\left\{x_{n}\right\}$ which is also a Parseval (or tight) frame. We show that the existence of a Parseval dual is equivalent to the problem whether $\left\{x_{n}\right\}$ can be dilated to an orthonormal basis (under an oblique projection). A necessary and sufficient condition for the existence of Parseval duals is obtained in terms of the frame excess. For a frame $\{\pi(g) \xi: g \in G\}$ induced by a projective unitary representation $\pi$ of a group $G$, it is possible that $\{\pi(g) \xi: g \in G\}$ can have a Parseval dual, but does not have a Parseval dual of the same type. The primary aim of this paper is to present a complete characterization for all the projective unitary representations $\pi$ such that every frame $\{\pi(g) \xi: g \in G\}$ (with a necessary lower frame bound condition) has a Parseval dual of the same type. As an application of this characterization together with a result about lattice tiling, we prove that every Gabor frame $\mathbf{G}(g, \mathcal{L}, \mathcal{K}$ ) (again with the same necessary lower frame bound condition) has a Parseval dual of the same type if and only if the volume of the fundamental domain of $\mathcal{L} \times \mathcal{K}$ is less than or equal to $\frac{1}{2}$.
\end{abstract}

\section{INTRODUCTION}

A frame for a Hilbert space $H$ is a sequence of vectors $\left\{x_{n}\right\} \subset H$ for which there exist constants $0<A \leq B<\infty$ such that, for every $x \in H$,

$$
A\|x\|^{2} \leq \sum\left|\left\langle x, x_{n}\right\rangle\right|^{2} \leq B\|x\|^{2} .
$$

The optimal constants (maximal for $A$ and minimal for $B$ ) are known respectively as the upper and lower frame bounds. A frame is called a tight frame if $A=B$, and is called a Parseval frame if $A=B=1$. If a sequence $\left\{x_{n}\right\}$ satisfies the upper bound condition in (1.1), then $\left\{x_{n}\right\}$ is also called a Bessel sequence. Frames are generalizations of Riesz bases and were first introduced by Duffin and Schaeffer [9] to deal with some difficult problems in nonharmonic Fourier analysis. The study of frame theory has drawn a lot of attention in recent years, partially because of its applications in signal processing as well as its close connections with other mathematical fields such as wavelet theory, time-frequency analysis, operator and operator algebra theory (cf. $[8,14,15,12,13,16,17,18,20,21,27,25,29]$ ).

Received by the editors February 22, 2005 and, in revised form, October 3, 2006

2000 Mathematics Subject Classification. Primary 42C15, 46C05, 47B10.

Key words and phrases. Frames, Parseval duals, frame representations, Gabor frames, lattice tiling.

(C) 2008 American Mathematical Society Reverts to public domain 28 years from publication 
For a Bessel sequence $\left\{x_{n}\right\}$, the analysis operator $\Theta$ is a bounded linear operator from $H$ to $\ell^{2}(\mathbb{N})$ defined by

$$
\Theta x=\sum_{n \in \mathbb{N}}\left\langle x, x_{n}\right\rangle e_{n},
$$

where $\left\{e_{n}\right\}$ is the standard orthonormal basis for $\ell^{2}(\mathbb{N})$. Clearly, $\left\{x_{n}\right\}$ is a frame for $H$ if and only if its analysis operator $\Theta$ is injective and has closed range. In this case, the operator $S:=\Theta^{*} \Theta: H \rightarrow H$ is invertible and is called the frame operator. It can be easily verified that

$$
\Theta^{*} e_{n}=x_{n}, \quad \forall n \in \mathbb{N}
$$

and hence

$$
S x=\sum_{n \in \mathbb{N}}\left\langle x, x_{n}\right\rangle x_{n}, \quad \forall x \in H .
$$

This implies that the lower frame bound is $\frac{1}{\left\|S^{-1}\right\|}$ and the upper frame bound is $\|S\|$. From (1.2), we also obtain a reconstruction formula:

$$
x=\sum_{n \in \mathbb{N}}\left\langle x, S^{-1} x_{n}\right\rangle x_{n}, \quad \forall x \in H .
$$

The frame $\left\{S^{-1} x_{n}\right\}$ is called the canonical dual of $\left\{x_{n}\right\}$. In general, if a frame $\left\{y_{n}\right\}$ satisfies

$$
x=\sum_{n \in \mathbb{N}}\left\langle x, y_{n}\right\rangle x_{n}, \quad \forall x \in H,
$$

then $\left\{y_{n}\right\}$ is called an alternate dual frame of $\left\{x_{n}\right\}$. The canonical and alternate duals are simply referred to as duals. It is well known that a frame has a unique dual if and only if it is a Riesz basis (cf. [23]).

Parseval frames have many nice features. In particular, if $\left\{x_{n}\right\}$ is a Parseval frame, then the canonical dual is itself, which of course is a Parseval frame. This paper is primarily concerned with the existence problem for a general frame or a frame with special structures to have a dual frame which is also a Parseval frame. Such a dual will be called a Parseval dual. We first note that not every frame has a Parseval dual. For instance, if a frame $\left\{x_{n}\right\}$ is a Riesz basis but not Parseval, then its dual (which is unique) also fails to be Parseval. Moreover, if $\left\{y_{n}\right\}$ is a Parseval dual for a frame $\left\{x_{n}\right\}$, then we have for every $x \in H$ that

$$
\begin{aligned}
\|x\|^{2} & =\sum_{n \in \mathbb{N}}\left\langle x, y_{n}\right\rangle\left\langle x_{n}, x\right\rangle \\
& \leq\left(\sum_{n \in \mathbb{N}}\left|\left\langle x, y_{n}\right\rangle\right|^{2}\right)^{1 / 2}\left(\sum_{n \in \mathbb{N}}\left|\left\langle x_{n}, x\right\rangle\right|^{2}\right)^{1 / 2} \\
& =\|x\| \cdot\left(\sum_{n \in \mathbb{N}}\left|\left\langle x, x_{n}\right\rangle\right|^{2}\right)^{1 / 2},
\end{aligned}
$$

which implies that

$$
\|x\|^{2} \leq \sum_{n \in \mathbb{N}}\left|\left\langle x, x_{n}\right\rangle\right|^{2}
$$


Thus a necessary condition for a frame to have a Parseval dual is that the lower frame bound is greater than or equal to one. This leads to a natural question:

Problem 1. Assume that the frame operator $S$ for a frame $\left\{x_{n}\right\}$ has the lower frame bound $\geq 1$. Under what conditions does $\left\{x_{n}\right\}$ admit a Parseval dual?

When the lower frame bound is strictly greater than 1 , the above question has been answered in [23]:

Proposition $1.1([23])$. Let $\left\{x_{n}\right\}$ be a frame for a Hilbert space $H$ with lower frame bound $>1$. Then $\left\{x_{n}\right\}$ has a Parseval dual if and only if the dimension of $\Theta(H)^{\perp}$ is greater than or equal to the dimension of $H$, where $\Theta$ is the analysis operator of $\left\{x_{n}\right\}$.

The dimension of $\Theta(H)^{\perp}$ is also called the excess [5] of $\left\{x_{n}\right\}$. We remark that the above excess condition is also sufficient when the lower frame bound is 1 . However, it is not a necessary condition in general. For example, any Parseval frame has itself as a Parseval dual. But the excess of a Parseval frame is not necessarily greater than or equal to $\operatorname{dim} H$. In section 2 we will establish the connection between the frame dilations and the existence of Parseval duals, from which we will obtain a complete answer to Problem 1 (Proposition 2.4).

Our main focus will be on those frames with special structures since most of the useful frames in theory and in applications are of this kind (typical examples include Gabor frames and wavelet frames). Therefore, it is natural to ask the question when do we have a Parseval dual with the same structure as the given frame. Our particular interest is in those frames induced by a projective unitary representation of group $G$. This particular class of frames has close connections with operator algebra theory and has many applications such as in time-frequency analysis, wavelet analysis and shift invariant subspace theory (cf. [16, 17, 18, 22]).

Recall that a projective unitary representation $\pi$ for a countable discrete (not necessarily abelian) group $G$ is a mapping $g \rightarrow \pi(g)$ from $G$ into the set of unitary operators on a Hilbert space $H_{\pi}$ such that $\pi(g) \pi(h)=\mu(g, h) \pi(g h)$ for all $g, h \in G$, where $\mu(g, h)$ belongs to the circle group $\mathbb{T}$. The mapping $(g, h) \rightarrow \mu(g, h)$ is then called a multiplier of $\pi$ (cf. [31]). The image of a projective unitary representation is also called a group-like unitary system since it resembles in many ways a group of unitary operators. A frame projective unitary representation is a projective unitary representation $\pi$ on $H_{\pi}$ such that there exists a vector $\eta \in H_{\pi}$ such that $\{\pi(g) \eta: g \in G\}$ is a frame for $H_{\pi}$. We will point out in section 3 that, in most cases, if $\{\pi(g) \eta: g \in G\}$ is a frame but not a Riesz basis, then it also has a Parseval dual frame which is not necessarily in the form of $\{\pi(g) \xi: g \in G\}$. Indeed, there exist many such frames which do not admit a Parseval dual of the same type (see Corollary 3.4). This leads us to the second question:

Problem 2. Under what condition on the projective unitary frame-representation $\pi$ of a group $G$ does every frame $\{\pi(g) \eta\}$ have a Parseval dual frame of the same type?

One of our main results of this paper is to answer the above question. Recall that two Bessel sequences $\left\{x_{n}\right\}$ and $\left\{y_{n}\right\}$ are said to be orthogonal if the range spaces of their analysis operators are orthogonal in $\ell^{2}(\mathbb{N})$. The concept of orthogonal frames (also called strongly disjoint frames) was introduced and systematically studied by R. Balan in [3, 4], D. Han and D. Larson in [23], and has been used in the 
investigation of orthogonal Weyl-Heisenberg frames, super wavelets and sampling (cf. $[1,10,11,32])$. We say that a projective unitary representation $\pi$ has frame multiplicity $k$ if $k$ is the largest number such that there exist $k$ mutually orthogonal frames $\left\{\pi(g) \xi_{i}\right\}(i=1, \ldots, k)$ for $H$. We will prove:

Theorem 1.2. Let $G$ be a group and let $\pi$ be a projective unitary frame representation of $G$. Then the following are equivalent:

(i) $\pi$ has frame multiplicity greater than or equal to 2 ;

(ii) every frame $\{\pi(g) \xi\}$ with the lower frame bound greater than or equal to 1 has a Parseval dual of the same type.

We apply the above theorem to Gabor frames. Let $\mathcal{L}$ and $\mathcal{K}$ be two full rank lattices in $\mathbb{R}^{d}$. A Gabor frame $\mathbf{G}(g, \mathcal{L}, \mathcal{K})$ (the definition of Gabor frames will be given in section 4 ) is a special type of frame induced by a projective unitary representation of the group $G=\mathcal{L} \times \mathcal{K}$ in $\mathbb{R}^{2 d}$ on $H=L^{2}\left(\mathbb{R}^{d}\right)$. By using the above theorem and a deep result on lattice tiling (Theorem 4.4) we obtain:

Theorem 1.3. Let $\mathcal{L}=A \mathbb{Z}^{d}$ and $\mathcal{K}=B \mathbb{Z}^{d}$ be two full-rank lattices in $\mathbb{R}^{d}$. Then the following are equivalent:

(i) every Gabor frame $\mathbf{G}(g, \mathcal{L}, \mathcal{K})$ with the lower frame bound $\geq 1$ has a Parseval dual of the form $\mathbf{G}(h, \mathcal{L}, \mathcal{K})$,

(ii) $|\operatorname{det}(A B)| \leq \frac{1}{2}$.

The rest of this paper is organized as follows. In section 2 we discuss the connection between the dilation property of frames and the existence problem for Parseval duals. In particular we give a necessary and sufficient condition for the existence of a Parseval dual. Sections 3 and 4 are devoted to proving the two main theorems and to discussing some results related to frame representations and Gabor frames. An essential part in the proof of Theorem 1.3 is a result about lattice tiling in $\mathbb{R}^{d}$ which has an independent interest to us. The proof of this tiling result will be given in the appendix section.

\section{Parseval DUAls AND Dilations}

There is a natural connection between the existence of the Parseval dual and the so-called dilation theorem of frames. Clearly, the image of an orthonormal basis under an orthogonal projection is a Parseval frame for the image space. The converse of this is also very well known in frame theory (cf. [23]):

Proposition 2.1. Let $\left\{x_{n}\right\}$ be a Parseval frame for a Hilbert space $H$. Then there exist a Hilbert space $\mathcal{K} \supseteq H$ and an orthonormal basis $\left\{e_{n}\right\}$ for $\mathcal{K}$ such that $x_{n}=P e_{n}$, where $P$ is the orthogonal projection from $\mathcal{K}$ onto $H$.

In other words, Parseval frames are exactly the orthogonal compressions of orthonormal bases. What about general frames? Is it true that we can "dilate" any general frame to an orthonormal basis? (By "dilate" here we mean that the frame is the image of an orthonormal basis under an oblique projection, which is an idempotent operator but not necessarily orthogonal.) However, this is not true in general; for example, if $\left\{x_{n}\right\}$ is a Riesz basis but not a tight frame, then it can never be the image of an orthonormal basis under an oblique projection. For some more detailed discussion on this problem we refer to [7] and a more recent paper [2]. The following theorem tells us that the dilation result holds for a frame $\left\{x_{n}\right\}$ if and only if $\left\{x_{n}\right\}$ has a Parseval dual. 
Theorem 2.2. Let $\left\{x_{n}\right\}$ be a frame for a Hilbert space $H$. Then the following statements are equivalent:

(i) $\left\{x_{n}\right\}$ has a Parseval dual,

(ii) there exists a Hilbert space $\mathcal{K} \supset H$, an orthonormal basis $\left\{f_{n}\right\}$ for $\mathcal{K}$ and an oblique projection $Q$ such that $Q f_{n}=x_{n}$ and $Q(\mathcal{K})=H$.

Proof. $(i) \Rightarrow(i i)$ : Let $\left\{y_{n}\right\}$ be a Parseval dual for $\left\{x_{n}\right\}$. Then by Proposition 2.1 there exist a Hilbert space $\mathcal{K} \supset H$ and an orthonormal basis $\left\{f_{n}\right\}$ for $\mathcal{K}$ such that $P f_{n}=y_{n}$, where $P$ is the orthogonal projection from $\mathcal{K}$ onto $H$. Define $Q$ on $\mathcal{K}$ by

$$
Q u=\sum_{n \in \mathbb{N}}\left\langle u, f_{n}\right\rangle x_{n}, \quad \forall u \in \mathcal{K} .
$$

Then $Q f_{n}=\sum_{m \in \mathbb{N}}\left\langle f_{n}, f_{m}\right\rangle x_{m}=x_{n}$ and for every $x \in H$ we have

$$
\begin{aligned}
Q x & =\sum_{n \in \mathbb{N}}\left\langle x, f_{n}\right\rangle x_{n} \\
& =\sum_{n \in \mathbb{N}}\left\langle x, P f_{n}\right\rangle x_{n} \\
& =\sum_{n \in \mathbb{N}}\left\langle x, y_{n}\right\rangle x_{n}=x .
\end{aligned}
$$

Clearly $Q(\mathcal{K}) \subseteq \mathcal{H}$, and hence $Q(\mathcal{K})=\mathcal{H}$. Moreover, $Q^{2} u=Q(Q u)=Q u$ since $Q u \in H$. Thus $Q^{2}=Q$, as required.

$(i i) \Rightarrow(i)$ : Assume $(i i)$ and let $y_{n}=P Q^{*} f_{n}$, where again $P$ is the orthogonal projection from $\mathcal{K}$ onto $H$. Then clearly $y_{n} \in H$. Note that for each $x \in H$ we have $Q^{2} x=P x=x$. Thus

$$
\begin{aligned}
x & =Q^{2} P x=\sum_{n \in \mathbb{N}}\left\langle Q P x, f_{n}\right\rangle f_{n} \\
& =\sum_{n \in \mathbb{N}}\left\langle x, P Q^{*} f_{n}\right\rangle Q f_{n} \\
& =\sum_{n \in \mathbb{N}}\left\langle x, y_{n}\right\rangle x_{n},
\end{aligned}
$$

and

$$
\begin{aligned}
\|x\|^{2} & =\sum_{n \in \mathbb{N}}\left|\left\langle x, f_{n}\right\rangle\right|^{2} \\
& =\sum_{n \in \mathbb{N}}\left|\left\langle Q x, f_{n}\right\rangle\right|^{2} \\
& =\sum_{n \in \mathbb{N}}\left|\left\langle x, Q^{*} f_{n}\right\rangle\right|^{2} \\
& =\sum_{n \in \mathbb{N}}\left|\left\langle x, P Q^{*} f_{n}\right\rangle\right|^{2} \\
& =\sum_{n \in \mathbb{N}}\left|\left\langle x, y_{n}\right\rangle\right|^{2} .
\end{aligned}
$$

So $\left\{y_{n}\right\}$ is a Parseval dual for $\left\{x_{n}\right\}$. 
Combining the above result with Proposition 1.1 we immediately have:

Corollary 2.3. Let $\left\{x_{n}\right\}$ be a frame for a Hilbert space $H$, and let $\Theta$ be its analysis operator. If $\left\|\left(\Theta^{*} \Theta\right)^{-1}\right\|<1$ (i.e. the lower frame bound is $>1$ ), then the following are equivalent:

(i) $\left\{x_{n}\right\}$ has a Parseval dual,

(ii) there exist a Hilbert space $\mathcal{K} \supset H$, an orthonormal basis $\left\{f_{n}\right\}$ for $\mathcal{K}$ and an oblique projection $Q$ such that $Q f_{n}=x_{n}$ and $Q(\mathcal{K})=H$,

(iii) the excess of $\left\{x_{n}\right\}$ is greater than or equal to $\operatorname{dim} H$.

As we pointed out in the introduction section, the above corollary is no longer true when the lower frame bound is 1 . The following takes care of the general case (i.e., we don't require $\left.\left\|\left(\Theta^{*} \Theta\right)^{-1}\right\|<1\right)$ :

Proposition 2.4. Let $\left\{x_{n}\right\}$ be a frame for a Hilbert space $H$, and let $\Theta$ be its frame operator. Assume that $\left\|\left(\Theta^{*} \Theta\right)^{-1}\right\| \leq 1$.

(i) If the excess of $\left\{x_{n}\right\}$ is $\infty$, then $\left\{x_{n}\right\}$ always has a Parserval dual.

(ii) If the excess of $\left\{x_{n}\right\}$ is finite, then $\left\{x_{n}\right\}$ has a Parserval dual if and only if $\operatorname{dim} R\left(\Theta^{*} \Theta-I\right)$ is less than or equal to the excess of $\left\{x_{n}\right\}$.

Proof. (i) Let $S=\Theta^{*} \Theta$. Since $\operatorname{dim}\left(\Theta(H)^{\perp}\right) \geq \operatorname{dim} H$, we can choose a closed subspace $N$ of $\Theta(H)^{\perp}$ such that $\operatorname{dim} N=\operatorname{dim} H$. Let $W: N \rightarrow H$ be a fixed unitary operator, and let $w_{n}=W Q e_{n}$, where $Q$ is the orthogonal projection from $\ell^{2}(\mathbb{N})$ onto $N$. Then $\left\{w_{n}\right\}$ is a Parseval frame for $H$, which is orthogonal with $\left\{x_{n}\right\}$.

Let $B=\sqrt{I-S^{-1}}$. Then $\left\{B w_{n}\right\}$ is also orthogonal with $\left\{x_{n}\right\}$. Let $T$ be the analysis operator for $\left\{S^{-1} x_{n}+B w_{n}\right\}$. Then

$$
\mathbb{T}^{*} T=S^{-1}+B^{*} B=I,
$$

where we used the orthogonality between $\left\{B w_{n}\right\}$ and $\left\{x_{n}\right\}$. Hence $\left\{S^{-1} x_{n}+B w_{n}\right\}$ is a Parseval frame for $H$. Moreover, for every $x \in H$, we have that

$$
\begin{aligned}
\sum_{n}\left\langle x, S^{-1} x_{n}+B w_{n}\right\rangle x_{n} & =\sum_{n}\left\langle x, x_{n}\right\rangle x_{n}+\sum_{n}\left\langle x, B w_{n}\right\rangle x_{n} \\
& =x+0=x .
\end{aligned}
$$

Therefore $\left\{S^{-1} x_{n}+B w_{n}\right\}$ is a Parseval dual for $\left\{x_{n}\right\}$.

(ii) follows from Theorem 3.4 in [2] and Theorem 2.2.

\section{Proof of Theorem 1.2}

From the previous section we have seen that the excess of a frame is associated with the existence of a Parseval dual. So we first take a close look at the excess of the frames associated with projective unitary representations.

Proposition 3.1. Let $G$ be a group such that $G$ contains an element $g_{0}$ of order $\infty$, and let $\pi$ be a projective unitary representation of $G$. If $\{\pi(g) \xi: g \in G\}$ is a frame (but not a Riesz basis) for $H_{\pi}$, then the excess of $\{\pi(g) \xi: g \in G\}$ is $\infty$. In particular, $\{\pi(g) \xi: g \in G\}$ has a Parseval dual when its lower frame bound is $\geq 1$.

Proof. Let $\Theta$ be the analysis operator of $\{\pi(g) \xi\}$ from $H_{\pi}$ to $\ell^{2}(G)$, and let $M=$ $\left(\Theta\left(H_{\pi}\right)\right)^{\perp}$. For each $g \in G$, the left regular representation $\lambda$ associated with $\mu(\cdot, \cdot)$ 
is defined by

$$
\lambda(g)\left(\chi_{h}\right)=\overline{\mu(g, h)} \chi_{g h}, \quad h \in G,
$$

where $\left\{\chi_{g}: g \in G\right\}$ is the standard orthonormal basis for $\ell^{2}(G)$ and $\mu(\cdot, \cdot)$ is the multiplier of $\pi$. Then $\lambda(g)$ is unitary.

Since $\pi$ is a projective unitary representation, it can be easily verified that $\Theta\left(H_{\pi}\right)$ is invariant under all the operators $\lambda(g)$, and so is $M$. In particular $M$ is invariant under $\lambda\left(g_{0}\right)$. Since $g_{0}$ has order $\infty$, we have that $\lambda\left(g_{0}\right)$ has no eigenvalue (i.e. point spectrum). Thus $\operatorname{dim} M=\infty$ since, otherwise, the restriction of $\lambda\left(g_{0}\right)$ to $M$ would have an eigenvalue. So the excess of $\{\pi(g) \xi\}$ is $\infty$.

The idea of proving that the excess is infinite in the above proposition has been used for general frames in [5] by looking at the point spectrum of intertwining operators. In particular, it was proved in [5] that Gabor and wavelet frames all have infinite excess if they are not Riesz bases. Note that Gabor frames (resp. wavelet frames) are frames induced by an ordered product of two unitary operator groups. With a slight modification of the proof, the above proposition can be generalized to the following result, which obviously covers the Gabor and wavelet frame cases.

Corollary 3.2. Let $\pi_{1}, \ldots, \pi_{k}$ be projective unitary representations for the groups $G_{1}, \ldots, G_{k}$, respectively, on the same representation Hilbert space $H$. Assume that $G_{1}$ contains an element $g_{0}$ of order $\infty$. If $\left\{\pi_{1}\left(g_{1}\right) \ldots \pi_{k}\left(g_{k}\right) \xi: g_{i} \in G_{i}\right\}$ is a frame for $H$ but not a Riesz basis, then the excess of $\left\{\pi_{1}\left(g_{1}\right) \ldots \pi_{k}\left(g_{k}\right) \xi: g_{i} \in G_{i}\right\}$ is $\infty$.

Our next result points out that in general a frame $\{\pi(g) \xi: g \in G\}$ (with the lower frame bound $\geq 1$ ) does not have a Parseval dual of the same type. We say that a frame $\{\pi(g) \xi: g \in G\}$ has a unique dual of the same type if $\{\pi(g) \xi: g \in G\}$ has only one dual of the form $\{\pi(g) \eta\}$. We need the following characterization result for uniqueness of the dual of the same type [18]:

Theorem 3.3. Let $G$ be a group and $\pi$ be a projective unitary representation of $G$. If $\{\pi(g) \xi\}$ is a Parseval frame for $H_{\pi}$, then $\{\pi(g) \xi\}$ has a unique dual of the same type if and only if

$$
\left\langle\pi\left(g_{1}\right) \pi\left(g_{2}\right) \xi, \xi\right\rangle=\left\langle\pi\left(g_{2}\right) \pi\left(g_{1}\right) \xi, \xi\right\rangle
$$

holds for all $g_{1}, g_{2} \in G$.

Corollary 3.4. Let $\pi$ be a unitary representation of an abelian group $G$. If $\{\pi(g) \xi\}$ is a frame for $H_{\pi}$, then it has a unique dual of the same type. In particular, if $\{\pi(g) \xi\}$ is not a Parseval frame, then it has no Parseval dual of the same type.

Proof. Let $S$ be the frame operator for $\{\pi(g) \xi\}$. Then $S \pi(g)=\pi(g) S$ for all $g \in G$. Suppose that, besides the canonical dual $\left\{\pi(g) S^{-1} \xi\right\},\{\pi(g) \eta\}$ is another dual frame of $\{\pi(g) \xi\}$. Then

$$
x=\sum_{g \in G}\langle x, \pi(g) \eta\rangle \pi(g) \xi
$$


for every $x \in H_{\pi}$. So

$$
\begin{aligned}
x & =S^{-1 / 2}\left(S^{1 / 2} x\right) \\
& =S^{-1 / 2} \sum_{g \in G}\left\langle S^{1 / 2} x, \pi(g) \eta\right\rangle \pi(g) \xi \\
& =\sum_{g \in G}\left\langle x, S^{1 / 2} \pi(g) \eta\right\rangle S^{-1 / 2} \pi(g) \xi \\
& =\sum_{g \in G}\left\langle x, \pi(g) S^{1 / 2} \eta\right\rangle \pi(g) S^{-1 / 2} \xi .
\end{aligned}
$$

Hence $\left\{\pi(g) S^{1 / 2} \eta\right\}$ is a dual frame of the Parseval frame $\left\{\pi(g) S^{-1 / 2} \xi\right\}$.

Since $G$ is abelian, we have that $\pi\left(g_{1}\right) \pi\left(g_{2}\right)=\pi\left(g_{1} g_{2}\right)=\pi\left(g_{2} g_{1}\right)=\pi\left(g_{2}\right) \pi\left(g_{1}\right)$. Thus the condition (3.4) holds for the Parseval frame $\left\{\pi(g) S^{-1 / 2} \xi\right\}$. So, from Theorem 3.3, $\left\{\pi(g) S^{-1 / 2} \xi\right\}$ has a unique dual of the same type. Therefore we have

$$
S^{1 / 2} \eta=S^{-1 / 2} \xi
$$

which implies that $\eta=S^{-1} \xi$. Hence $\{\pi(g) \xi\}$ has a unique dual of the same type. Combining this with the fact that $\{\pi(g) \xi\}$ is Parseval if and only if $\left\{S^{-1} \pi(g) \xi\right\}$ is Parseval, we obtain the second part of the corollary

To prove Theorem 1.2, we need to introduce a few more concepts. We first recall some notation in von Neumann algebra theory (cf. [28]). Let $B(H)$ be the algebra of all bounded linear operators on a separable Hilbert space $H$. A $*$-subalgebra $\mathcal{M}$ of $B(H)$ is a subalgebra with the property that $T^{*}$ belongs to $\mathcal{M}$ whenever $T \in \mathcal{M}$. A $*$-subalgebra $\mathcal{M}$ is called a von Neumann algebra if the identity operator $I$ is in $\mathcal{M}$ and if it is closed in the weak operator topology. A von Neumann algebra is called finite if every isometry in the algebra is unitary. Two orthogonal projections $P$ and $Q$ in a von Neumann algebra $\mathcal{M}$ are said to be equivalent if there exists a partial isometry $T \in \mathcal{M}$ such that $T T^{*}=P$ and $T^{*} T=Q$.

Let $M$ be an invariant subspace of a projective unitary representation $\pi$. Then the restriction of $\pi$ to $M$ is also a projective unitary representation of $G$ with the same multiplier, and this representation is called a subrepresentation of $\pi$, and is denoted by $\pi_{P}$, where $P$ is the orthogonal projection from $H_{\pi}$ onto $M$. Two projective unitary representations $\pi$ and $\sigma$ are said to be unitarily equivalent if there exists a unitary operator $U$ from $H_{\pi}$ onto $H_{\sigma}$ such that

$$
U \pi(g)=\sigma(g) U
$$

holds for every $g \in G$.

Let $\pi$ be a projective unitary representation of a group $G$ with multiplier $\mu$. Recall that the left regular unitary representation $\lambda$ of $G$ on $\ell^{2}(G)$ associated with $\mu$ is defined by

$$
\lambda(g) \chi_{h}=\overline{\mu(g, h)} \chi_{g h}, \quad h \in G,
$$

where $\left\{\chi_{g}: g \in G\right\}$ is the standard orthonormal basis for $\ell^{2}(G)$. Then it can be checked that $\lambda$ is a projective unitary representation of $G$ with the same multiplier $\mu$. Let $\lambda(G)^{\prime}:=\left\{T \in B\left(\ell^{2}(G)\right): T \lambda(g)=\lambda(g) T, g \in G\right\}$ be the commutant of $\lambda(G)$. Then $\lambda(G)^{\prime}$ is a finite von Neumann algebra. For any orthogonal projection $P \in \lambda(G)^{\prime}$, the restriction of $\lambda$ to $M:=P\left(\ell^{2}(G)\right)$ is always a frame projective unitary representation since $M$ is invariant under $\lambda$. It is evident that if we let 
$\eta=P \chi_{e}$, then $\left\{\lambda_{P}(g) \eta\right\}$ is a Parseval frame for $M$. That is, every subrepresentation of $\lambda$ is a frame representation. In fact, the converse is also true:

Lemma 3.5 ([16]). If $\pi$ is a frame projective unitary representation, then $\pi$ is unitarily equivalent to a subrepresentation of the associated left regular representation.

Lemma 3.6. Let $\pi$ be a projective unitary representation of a group $G$ and $Q$ an orthogonal projection in $\lambda(G)^{\prime}$. Assume that $\{\pi(g) \eta\}$ is a Parseval frame for $H_{\pi}$ and $P$ is the orthogonal projection from $\ell^{2}(G)$ onto $\Theta_{\eta}\left(H_{\pi}\right)$. Then the following are equivalent:

(i) $Q \backsim P$ in $\lambda(G)^{\prime}$,

(ii) there exists a Parseval frame $\{\pi(g) \xi\}$ for $H_{\pi}$ such that $Q$ is the orthogonal projection from $\ell^{2}(G)$ onto $\Theta_{\xi}\left(H_{\pi}\right)$.

Proof. From Lemma 3.5, without loss of the generality, we can assume that $\pi=\lambda_{P}$, $H_{\pi}=P\left(\ell^{2}(G)\right)$, and $\eta=P \chi_{e}$.

First assume that $Q \backsim P$ in $\lambda(G)^{\prime}$. Then there exists a partial isometry $V \in$ $\lambda(G)^{\prime}$ such that $V^{*} V=P$ and $V V^{*}=Q$. Let $\xi=V^{*} \chi_{e} \in H_{\pi}=P\left(\ell^{2}(G)\right)$, where $e$ is the unit of $G$. Thus, for any $x \in H_{\pi}$, we have

$$
\begin{aligned}
\sum_{g \in G}|\langle x, \pi(g) \xi\rangle|^{2} & =\sum_{g \in G}\left|\left\langle x, \lambda_{P}(g) \xi\right\rangle\right|^{2} \\
& =\sum_{g \in G}\left|\left\langle x, \lambda(g) P V^{*} \chi_{e}\right\rangle\right|^{2} \\
& =\sum_{g \in G}\left|\left\langle x, \lambda(g) V^{*} \chi_{e}\right\rangle\right|^{2} \\
& =\sum_{g \in G}\left|\left\langle V x, \lambda(g) \chi_{e}\right\rangle\right|^{2} \\
& =\sum_{g \in G}\left|\left\langle V x, \chi_{g}\right\rangle\right|^{2} \\
& =\|V x\|^{2}=\|x\|^{2},
\end{aligned}
$$

where in the last equality we use the fact that $V$ is isometric on $P\left(\ell^{2}(G)\right)$. So $\{\pi(g) \xi\}$ is a Parseval frame for $H$. Similarly, for each $x \in H_{\pi}$ we also have

$$
\begin{aligned}
\sum_{g \in G}\langle x, \pi(g) \xi\rangle \chi_{g} & =\sum_{g \in G}\left\langle V x, \lambda(g) \chi_{e}\right\rangle \chi_{g} \\
& =\sum_{g \in G}\left\langle V x, \chi_{g}\right\rangle \chi_{g} .
\end{aligned}
$$

Thus $\Theta_{\xi}(H)=V H=Q\left(\ell^{2}(G)\right)$, as expected.

Conversely, assume that $\{\pi(g) \xi\}$ is a Parseval frame for $H_{\pi}=P\left(\ell^{2}(G)\right)$ such that $Q$ is the orthogonal projection from $\ell^{2}(G)$ onto $\Theta_{\xi}\left(H_{\pi}\right)$. Then $Q \in \lambda(G)^{\prime}$. We define a linear operator $V$ on $\ell^{2}(G)$ by letting $V=\Theta_{\xi}$ on $H_{\pi}=P\left(\ell^{2}(G)\right)$ and $V x=0$ when $x$ is orthogonal to $P\left(\ell^{2}(G)\right)$. Clearly $V$ is a partial isometry satisfying $V V^{*}=Q$ and $V^{*} V=P$. Moreover, it is an easy exercise to verify that $V$ commutes with all $\lambda(g)$ for every $g \in G$. Hence, $Q \backsim P$ in $\lambda(G)^{\prime}$.

Lemma 3.7. Let $G$ be a group and let $\pi$ be a projective unitary representation of $G$ with frame multiplicity greater than or equal to 2 . Then for every frame $\{\pi(g) \eta\}$ 
for $H$, there exists a Parseval frame $\{\pi(g) \xi\}$ such that $\{\pi(g) \xi\}$ and $\{\pi(g) \eta\}$ are orthogonal.

Proof. Since $\pi$ has multiplicity at least 2, there exist two frames $\{\pi(g) x: g \in G\}$ and $\{\pi(g) y: g \in G\}$ for $H$ that are orthogonal. Let $P$ and $Q$ be the orthogonal projections onto $\Theta_{x}(H)$ and $\Theta_{y}(H)$, respectively. Then $P, Q \in \lambda(G)^{\prime}$ and $P \perp Q$. By Lemma 3.6, we also have $P \backsim Q$.

Let $\{\pi(g) \eta\}$ be a frame for $H$, and $R$ be the orthogonal projections onto $\Theta_{\eta}(H)$. Then, again by Lemma 3.6, we have $R \backsim P \backsim Q$. Since $\lambda(G)^{\prime}$ is a finite Neumann algebra, it follows from the property of finite von Neumann algebras (cf. [28]) that $R^{\perp} \backsim P^{\perp}$. Note that $Q$ is a subprojection of $P^{\perp}$. Thus, there exists a subprojection $D$ of $R^{\perp}$ such that $D$ is equivalent to $Q$ in $\lambda(G)^{\prime}$. Thus, from Lemma 3.6, there exists a frame $\{\pi(g) \xi\}$ for $H$ such that $D$ is the orthogonal projection from $\ell^{2}(G)$ onto $\Theta_{\xi}\left(\mathcal{H}_{\pi}\right)$. Since $D$ and $R$ are orthogonal, it follows that $\{\pi(g) \eta\}$ and $\{\pi(g) \xi\}$ are orthogonal.

Now we are ready to prove Theorem 1.2.

Proof of Theorem 1.2. $(i) \Rightarrow(i i)$ : Assume that $\pi$ has frame multiplicity greater than or equal to 2. Let $\{\pi(g) \xi\}$ be a frame for $H_{\pi}$, and $S=\Theta_{\xi}^{*} \Theta_{\xi}$ be its frame operator with $\left\|S^{-1}\right\| \leq 1$. Then $I-S^{-1}$ is a positive operator on $H_{\pi}$. Let $B=$ $\sqrt{I-S^{-1}}$. We have $B \in \pi(G)^{\prime}$.

Since $\pi$ has frame multiplicity greater than or equal to 2, it follows from Lemma 3.7 that there exists a Parseval frame $\{\pi(g) \eta\}$ such that $\{\pi(g) \eta\}$ and $\left\{\pi(g) S^{-1} \xi\right\}$ are orthogonal. Let $\Theta_{\eta}$ be the analysis operator of $\{\pi(g) \eta\}$. Then we have $\Theta_{\eta}^{*} \Theta_{\eta}=I$ and $\Theta_{S^{-1} \xi}^{*} \Theta_{\eta}=0=\Theta_{\xi}^{*} \Theta_{\eta}$. Since $B \in \pi(G)^{\prime}$, we have that

$$
\begin{aligned}
B \Theta_{\eta}^{*} \Theta_{\eta} B x & =\sum_{g \in G}\langle B x, \pi(g) \eta\rangle B \pi(g) \eta \\
& =\sum_{g \in G}\langle x, B \pi(g) \eta\rangle B \pi(g) \eta \\
& =\sum_{g \in G}\langle x, \pi(g) B \eta\rangle \pi(g) B \eta \\
& =\Theta_{B \eta}^{*} \Theta_{B \eta} x
\end{aligned}
$$

holds for every $x \in H$, which implies that

$$
I-S^{-1}=B^{2}=B \Theta_{\eta}^{*} \Theta_{\eta} B=\Theta_{B \eta}^{*} \Theta_{B \eta} .
$$

Thus

$$
\Theta_{S^{-1} \xi}^{*} \Theta_{S^{-1} \xi}+\Theta_{B \eta}^{*} \Theta_{B \eta}=I .
$$

Let $\varphi=S^{-1} \xi+B \eta$. We claim that $\{\pi(g) \varphi\}$ is a Parseval dual of $\{\pi(g) \xi\}$. In fact, since $\{\pi(g) \eta\}$ and $\left\{\pi(g) S^{-1} \xi\right\}$ are orthogonal, we have that for every $x \in H$,

$$
\begin{aligned}
\Theta_{S^{-1} \xi}^{*} \Theta_{B \eta} x & =\sum_{g \in G}\langle x, \pi(g) B \eta\rangle \pi(g) S^{-1} \xi \\
& =\sum_{g \in G}\langle B x, \pi(g) \eta\rangle \pi(g) S^{-1} \xi \\
& =\Theta_{S^{-1} \xi}^{*} \Theta_{\eta} B x=0,
\end{aligned}
$$

and similarly, $\Theta_{\xi}^{*} \Theta_{B \eta}=0$. 
Therefore we have

$$
\begin{aligned}
\Theta_{\varphi}^{*} \Theta_{\varphi} & =\Theta_{S^{-1} \xi}^{*} \Theta_{S^{-1} \xi} \Theta_{S^{-1} \xi}^{*} \Theta_{B \eta}+\Theta_{B \eta}^{*} \Theta_{S^{-1} \xi}+\Theta_{B \eta}^{*} \Theta_{B \eta} x \\
& =\Theta_{S^{-1} \xi}^{*} \Theta_{S^{-1} \xi}+\Theta_{B \eta}^{*} \Theta_{B \eta}=I,
\end{aligned}
$$

and

$$
\Theta_{\varphi}^{*} \Theta_{\xi}=\Theta_{S^{-1} \xi}^{*} \Theta_{\xi}+\Theta_{B \eta}^{*} \Theta_{\xi}=I+0=I .
$$

Hence $\{\pi(g) \varphi\}$ is a Parseval dual of $\{\pi(g) \xi\}$.

$(i i) \Rightarrow(i)$ : Pick a frame $\{\pi(g) \xi\}$ for $H$ such that $\left\|S^{-1}\right\|<1$, where $S=\Theta_{\xi}^{*} \Theta_{\xi}$ is the frame operator. Then, by assumption, $\{\pi(g) \xi\}$ has a Parseval dual frame, say $\{\pi(g) \eta\}$. Let $h=\eta-S^{-1} \xi$. Then for every $x \in H$ we have

$$
\begin{aligned}
\sum_{g \in G}\langle x, \pi(g) h\rangle \pi(g) \xi & =\sum_{g \in G}\langle x, \pi(g) \eta\rangle \pi(g) \xi-\sum_{g \in G}\left\langle x, \pi(g) S^{-1} \xi\right\rangle \pi(g) \xi \\
& =x-x=0,
\end{aligned}
$$

which implies that $\{\pi(g) \xi\}$ and $\{\pi(g) h\}$ are orthogonal. Let $\Theta_{h}$ and $\Theta_{\eta}$ be the analysis operators for $\{\pi(g) h\}$ and $\{\pi(g) \eta\}$, respectively. Then we have

$$
\begin{aligned}
I & =\Theta_{\eta}^{*} \Theta_{\eta}=\Theta_{S^{-1} \xi}^{*} \Theta_{S^{-1} \xi}+\Theta_{S^{-1} \xi}^{*} \Theta_{h}+\Theta_{h}^{*} \Theta_{S^{-1} \xi}+\Theta_{h}^{*} \Theta_{h} \\
& =\Theta_{S^{-1} \xi}^{*} \Theta_{S^{-1} \xi}+\Theta_{h}^{*} \Theta_{h} \\
& =S^{-1} \Theta_{\xi}^{*} \Theta_{\xi} S^{-1}+\Theta_{h}^{*} \Theta_{h} \\
& =S^{-1}+\Theta_{h}^{*} \Theta_{h} .
\end{aligned}
$$

Thus $\Theta_{h}^{*} \Theta_{h}$ is invertible since $\left\|S^{-1}\right\|<1$. Therefore $\{\pi(g) h\}$ is also a frame for $H$. Now we have two orthogonal frames $\{\pi(g) \xi\}$ and $\{\pi(g) h\}$, which implies that $\pi$ has frame multiplicity at least 2 .

Corollary 3.8. Let $\pi$ be a frame projective unitary representation of $G$ such that every frame with the lower frame bound greater than or equal to 1 has a Parseval dual of the same type. Then $\|\xi\|^{2} \leq 1 / 2$ for every Parseval frame $\{\pi(g) \xi\}$.

Proof. Let $\{\pi(g) \xi\}$ be a Parseval frame for H. By Theorem 1.2 and Lemma 3.7, there exists a Parseval frame $\{\pi(g) \eta\}$ such that $\{\pi(g) \xi\}$ and $\{\pi(g) \eta\}$ are orthogonal frames. Then, by Theorem 2.9 in [23], $\{\pi(g) \xi \oplus \pi(g) \eta: g \in G\}$ is a Parseval frame for $H \oplus H$, which implies that $\|\xi \oplus \eta\| \leq 1$. We recall a fact [16] that $\|\varphi\|=\|\psi\|$ for any two Parseval frames $\{\pi(g) \varphi\}$ and $\{\pi(g) \psi\}$. Therefore we have $\|\xi\|^{2}=\|\eta\|^{2} \leq 1 / 2$.

For some special cases, the condition $\|\xi\|^{2} \leq 1 / 2$ for a Parseval frame $\{\pi(g) \xi\}$ is also sufficient for $\pi$ to have frame multiplicity at least 2 .

Corollary 3.9. Let $G$ be a group and $\pi$ be a frame projective unitary representation with multiplier $\mu$. Assume that for each $g \in G$ either $\left\{h^{-1} g h: h \in G\right\}$ is infinite or $\left\{\mu\left(h g, h^{-1}\right): h \in G\right\}$ is infinite. Then the following are equivalent:

(i) every frame $\{\pi(g) \xi\}$ with the lower frame bound greater than or equal to 1 has a Parseval dual of the same type.

(ii) $\|\xi\|^{2} \leq \frac{1}{2}$ for some Parseval frame $\{\pi(g) \xi\}$;

(iii) $\|\xi\|^{2} \leq \frac{1}{2}$ for every Parseval frame $\{\pi(g) \xi\}$.

In particular, this is true when $G$ is an infinite conjugate class (ICC) group. 
Proof. The implication $(i) \Rightarrow$ (iii) follows from Corollary 3.8. So it suffices to show that $(i i) \Rightarrow(i)$. By Theorem 1.2, we need to show that $(i)$ implies that $\pi$ has frame multiplicity at least 2 .

Let $\{\pi(g) \xi\}$ be a Parseval frame for $H$ with $\|\xi\| \leq \frac{1}{2}$. By Proposition 1.2 in [16], the von Neumann algebra $\lambda(G)^{\prime}$ is a finite factor, where $\lambda$ is the left regular projective unitary representation of $G$ associated with $\mu$. Let $P$ be the orthogonal projection from $\ell^{2}(G)$ onto the range space $\Theta_{\xi}(H)$ of the analysis operator $\Theta_{\xi}$ for the Parseval frame $\{\pi(g) \xi\}$. Then $\operatorname{tr}(P):=\left\langle P \chi_{e}, \chi_{e}\right\rangle=\|\xi\|^{2} \leq \frac{1}{2}$. Since $\lambda(G)^{\prime}$ is a finite factor von Neumann algebra, it follows that there exists a subprojection $Q$ of $P^{\perp}$ such that $P$ and $Q$ are equivalent in $\lambda(G)^{\prime}$. Hence, by Lemma 3.6, there exists a Parseval frame $\{\pi(g) \eta: g \in G\}$ such that $\theta_{\eta}(H)=\operatorname{Range}(Q)$. Therefore $\{\pi(g) \xi: g \in G\}$ and $\{\pi(g) \eta: g \in G\}$ are two orthogonal frames, which implies that $\pi$ has frame multiplicity at least 2 .

\section{Proof of Theorem 1.3}

In this section we prove our main result about Gabor frames. Let $\mathcal{L}$ and $\mathcal{K}$ be two full-rank lattices in $\mathbb{R}^{d}$, and let $g(x) \in L^{2}\left(\mathbb{R}^{d}\right)$. Then the Gabor family is the following family of functions in $L^{2}\left(\mathbb{R}^{d}\right)$ :

$$
\mathbf{G}(g, \mathcal{L}, \mathcal{K}):=\left\{e^{2 \pi i\langle\ell, x\rangle} g(x-\kappa) \mid \ell \in \mathcal{L}, \kappa \in \mathcal{K}\right\} .
$$

Such a family was first introduced by Gabor [19] in 1946 for signal processing and has recently become an active research topic in time-frequency analysis (cf. $[6,12,13,20])$. A Gabor family $\mathbf{G}(g, \mathcal{L}, \mathcal{K})$ is called a Gabor frame if $\mathbf{G}(g, \mathcal{L}, \mathcal{K})$ is a frame for $L^{2}\left(\mathbb{R}^{d}\right)$. Let $T_{t}$ and $E_{s}$ be the translation and modulation unitary operators defined by:

$$
T_{t} f(x)=f(x-t)
$$

and

$$
E_{s} f(x)=e^{2 \pi i\langle s, x\rangle} f(x)
$$

for all $f \in L^{2}\left(\mathbb{R}^{d}\right)$. Define $\pi: \Lambda \rightarrow B\left(L^{2}\left(\mathbb{R}^{d}\right)\right)$ by

$$
\pi(\ell, \kappa)=E_{\ell} T_{\kappa}, \quad \ell \in \mathcal{L}, \kappa \in \mathcal{K} .
$$

Then $\pi$ is a projective unitary representation of $\mathcal{L} \times \mathcal{K}$ on $H=L^{2}\left(\mathbb{R}^{d}\right)$. It is obvious that

$$
\mathbf{G}(g, \mathcal{L}, \mathcal{K})=\{\pi(\ell, \kappa) g: \ell \in \mathcal{L}, \kappa \in \mathcal{K}\} .
$$

Therefore, we call $\pi$ the Gabor projective unitary representation of $\mathcal{L} \times \mathcal{K}$.

Write $\mathcal{L}=A \mathbb{Z}^{d}$ and $\mathcal{K}=B \mathbb{Z}^{d}$ with $A$ and $B$ nonsingular $d \times d$ real matrices. The following is well known in Gabor theory (see [25]):

Lemma 4.1. Let $\mathcal{L}=A \mathbb{Z}^{d}$ and $\mathcal{K}=B \mathbb{Z}^{d}$. Then the Gabor projective unitary representation $\pi$ of $\mathcal{L} \times \mathcal{K}$ is a frame projective unitary representation if and only if $|\operatorname{det}(A B)| \leq 1$. Moreover, $|\operatorname{det}(A B)|=1$ if and only if every Gabor frame $\mathbf{G}(g, \mathcal{L}, \mathcal{K})$ is a Riesz basis.

Combining this with Proposition 3.1, we have

Proposition 4.2. Suppose that $|\operatorname{det}(A B)|<1$. Then every Gabor frame $\mathbf{G}(g, \mathcal{L}, \mathcal{K})$ with the lower frame bound $\geq 1$ has a Parseval dual (not necessarily a Gabor frame). 
To prove Theorem 1.3, we need to introduce the concept of tiling for $\mathbb{R}^{d}$. Let $\Omega$ be a measurable set in $\mathbb{R}^{d}$ (not necessarily bounded), and let $\mathcal{L}$ be a full rank lattice in $\mathbb{R}^{d}$. We say $\Omega$ tiles $\mathbb{R}^{d}$ by $\mathcal{L}$, or $\Omega$ is a fundamental domain of $\mathcal{L}$, if

(i) $\bigcup_{\ell \in \mathcal{L}}(\Omega+\ell)=\mathbb{R}^{d}$ a.e.;

(ii) $(\Omega+\ell) \cap\left(\Omega+\ell^{\prime}\right)$ has Lebesgue measure 0 for any $\ell \neq \ell^{\prime}$ in $\mathcal{L}$.

We say that $\Omega$ packs $\mathbb{R}^{d}$ by $\mathcal{L}$ if only (ii) holds. Equivalently, $\Omega$ tiles $\mathbb{R}^{d}$ by $\mathcal{L}$ if and only if

$$
\sum_{\ell \in \mathcal{L}} \chi_{\Omega}(x-\ell)=1 \text { for a.e. } x \in \mathbb{R}^{d},
$$

and $\Omega$ packs $\mathbb{R}^{d}$ by $\mathcal{L}$ if and only if

$$
\sum_{\ell \in \mathcal{L}} \chi_{\Omega}(x-\ell) \leq 1 \text { for a.e. } x \in \mathbb{R}^{d} .
$$

Let $\mathrm{v}(\mathcal{L})$ denote the volume of $\mathcal{L}$, i.e. $\mathrm{v}(\mathcal{L})=|\operatorname{det}(A)|$ for $\mathcal{L}=A \mathbb{Z}^{d}$. Clearly, $\mu(\Omega)=\mathrm{v}(\mathcal{L})$ if $\Omega$ tiles by $\mathcal{L}$, and $\mu(\Omega) \leq \mathrm{v}(\mathcal{L})$ if $\Omega$ packs by $\mathcal{L}$. Furthermore, if $\Omega$ packs $\mathbb{R}^{d}$ by $\mathcal{L}$ and $\mu(\Omega)=\mathrm{v}(\mathcal{L})$, then $\Omega$ necessarily tiles $\mathbb{R}^{d}$ by $\mathcal{L}$. The following is the main result obtained in [25] and was used to answer an open question for the existence of Gabor frames in higher dimensions $([25,26])$.

Theorem 4.3. Let $\mathcal{L}, \mathcal{K}$ be two full rank lattices in $\mathbb{R}^{d}$ such that $\mathrm{v}(\mathcal{L}) \geq \mathrm{v}(\mathcal{K})$. Then there exists a measurable set $\Omega$ in $\mathbb{R}^{d}$ such that $\Omega$ tiles $\mathbb{R}^{d}$ by $\mathcal{K}$ and packs $\mathbb{R}^{d}$ by $\mathcal{L}$.

The following modified version of the above theorem is essential in the proof of Theorem 1.3.

Theorem 4.4. Let $\mathcal{L}, \mathcal{K}$ be two full rank lattices in $\mathbb{R}^{d}$ such that $\mathrm{v}(\mathcal{L}) \geq 2 \mathrm{v}(\mathcal{K})$. Then there exist two measurable sets $\Omega$ and $E$ in $\mathbb{R}^{d}$ such that

(i) both $\Omega$ and $E$ tile $\mathbb{R}^{d}$ by $\mathcal{K}$ and pack $\mathbb{R}^{d}$ by $\mathcal{L}$;

(ii) $(\Omega+\ell) \cap\left(E+\ell^{\prime}\right)$ has Lebesgue measure 0 for any $\ell, \ell^{\prime}$ in $\mathcal{L}$.

We note that this result is trivial when $d=1$. In fact, let $\mathcal{L}=\alpha \mathbb{Z}$ and $\mathcal{K}=\beta \mathbb{Z}$ with $0<\alpha \beta \leq \frac{1}{2}$. Then $2 \beta \leq \frac{1}{\alpha}$. Let $\Omega=[0, \beta)$ and $E=[\beta, 2 \beta)$. Then it is obvious that $\Omega$ and $E$ satisfy both conditions $(i)$ and $(i i)$ in Theorem 4.4. However, the higher dimension case is very complicated due to the complexity of the lattices. By using several key lemmas proved in [25], the proof of Theorem 4.4 is similar to the proof of Theorem 4.3. Since the proof is very technical, we will give a sketch of the proof in the appendix section.

Lemma $4.5([16,25])$. Let $\mathcal{L}=A \mathbb{Z}^{d}, \mathcal{K}=B \mathbb{Z}^{d}$ be two full rank lattices in $\mathbb{R}^{d}$. If $\mathbf{G}(h, \mathcal{L}, \mathcal{K})$ is a Parseval frame for $L^{2}\left(\mathbb{R}^{d}\right)$, then $\|g\|^{2}=|\operatorname{det}(A B)|$.

Proof of Theorem 1.3. (i) $\Rightarrow($ ii $)$ : Assume that every Gabor frame $\mathbf{G}(g, \mathcal{L}, \mathcal{K})$ with the lower frame bound $\geq 1$ has a Parseval dual of the form $\mathbf{G}(h, \mathcal{L}, \mathcal{K})$. Then, by Corollary 3.9, $\|g\|^{2} \leq \frac{1}{2}$ for every Parseval frame $\mathbf{G}(g, \mathcal{L}, \mathcal{K})$. Thus, from Lemma $4.5,|\operatorname{det}(A B)|=\|g\|^{2} \leq \frac{1}{2}$.

$(i i) \Rightarrow(i)$ : By Theorem 1.2, we need to show that if $|\operatorname{det}(A B)| \leq 1 / 2$, then there exist two orthogonal Gabor frames $\mathbf{G}(g, \mathcal{L}, \mathcal{K})$ and $\mathbf{G}(h, \mathcal{L}, \mathcal{K})$.

Note that if we define $U: L^{2}\left(\mathbb{R}^{d}\right) \rightarrow L^{2}\left(\mathbb{R}^{d}\right)$ by

$$
(U f)(x)=\frac{1}{\sqrt{|\operatorname{det}(A)|}} f\left(\left(A^{t}\right)^{-1} x\right), \quad f \in L^{2}\left(\mathbb{R}^{d}\right),
$$


then $U$ is a unitary operator such that

$$
U E_{A m} T_{B n} U^{*}=E_{m} T_{A^{t} B n}, \quad m, n \in \mathbb{Z}^{d} .
$$

So without loss of generality, we can assume that $A=I$ and $|\operatorname{det} B| \leq \frac{1}{2}$.

By Theorem 4.4, there exist two measurable subsets $\Omega$ and $E$ of $\mathbb{R}^{d}$ such that they both tile $\mathbb{R}^{d}$ by $\mathcal{K}$ and pack $\mathbb{R}^{d}$ by $\mathbb{Z}^{d}$, and $(\Omega+m) \cap(E+n)$ has measure zero for all $m, n \in \mathbb{Z}^{d}$. Let $g=\chi_{\Omega}$ and $h=\chi_{E}$. We claim that $\mathbf{G}\left(g, \mathbb{Z}^{d}, \mathcal{K}\right)$ and $\mathbf{G}\left(h, \mathbb{Z}^{d}, \mathcal{K}\right)$ are two orthogonal Parseval frames for $L^{2}\left(\mathbb{R}^{d}\right)$.

To see that $\mathbf{G}\left(g, \mathbb{Z}^{d}, \mathcal{K}\right)$ is a Parseval frame for $L^{2}\left(\mathbb{R}^{d}\right)$, we note that for each $\kappa \in \mathcal{K},\left\{e^{2 \pi i\langle x, m\rangle} \chi_{\Omega+\kappa}: n \in \mathbb{Z}^{d}\right\}$ is a Parseval frame for $L^{2}(\Omega+\kappa)$ since $\Omega$ packs by $\mathbb{Z}^{d}$. Since $\Omega$ also tiles $\mathbb{R}^{d}$ by $\mathcal{K}$, it follows that

$$
\mathbf{G}\left(g, \mathbb{Z}^{d}, \mathcal{K}\right)=\left\{e^{2 \pi i\langle x, m\rangle} \chi_{\Omega+\kappa}: n \in \mathbb{Z}^{d}, \kappa \in \mathcal{K}\right\}
$$

is a Parseval frame for $L^{2}\left(\mathbb{R}^{d}\right)$ since $L^{2}\left(\mathbb{R}^{d}\right)=\bigoplus_{\kappa \in \mathcal{K}} L^{2}(\Omega+\kappa)$. For the same reason $\mathbf{G}\left(h, \mathbb{Z}^{d}, \mathcal{K}\right)$ is also a Parseval frame for $L^{2}\left(\mathbb{R}^{d}\right)$.

Now we check the orthogonality of the two frames. Let $Q$ be a fundamental domain of $\mathbb{Z}^{d}$ containing $E$. Let $\left\{\Omega_{k}\right\}$ be a partition of $\Omega$ such that $\Omega_{k}+n \subset Q$ for each $k \in \mathbb{Z}^{d}$ and set

$$
\tilde{\Omega}=\sum_{k \in \mathbb{Z}^{d}}\left(\Omega_{k}+k\right) .
$$

Then $\tilde{\Omega} \cap E$ has measure zero since $(\Omega+m) \cap(E+n)$ has measure zero for all $m, n \in \mathbb{Z}^{d}$. We first prove that

$$
\sum_{m \in \mathbb{Z}^{d}}\left\langle f, E_{m} g\right\rangle E_{m} h=0
$$

for all $f \in L^{2}\left(\mathbb{R}^{d}\right)$. Denote by $P_{F}$ the orthogonal projection from $L^{2}\left(\mathbb{R}^{d}\right)$ onto $L^{2}(F)$ for any measurable subset $F$ of $\mathbb{R}^{d}$, where we identify $L^{2}(F)$ with $\chi_{F} \cdot L^{2}\left(\mathbb{R}^{d}\right)$. Then

$$
\begin{aligned}
\sum_{m \in \mathbb{Z}^{d}}\left\langle f, E_{m} g\right\rangle E_{m} h & =\sum_{m \in \mathbb{Z}^{d}}\left(\int_{\Omega} f(x) e^{-2 \pi i\langle x, m\rangle} d x\right) e^{2 \pi i\langle x, m\rangle} \chi_{E} \\
& =\sum_{m \in \mathbb{Z}^{d}}\left(\sum_{k \in \mathbb{Z}^{d}} \int_{\Omega_{k}} f(x) e^{-2 \pi i\langle x, m\rangle} d x\right) e^{2 \pi i\langle x, m\rangle} \chi_{E} \\
& =\sum_{m \in \mathbb{Z}^{d}}\left(\sum_{k \in \mathbb{Z}^{d}} \int_{\Omega_{k}+k} f(x-k) e^{-2 \pi i\langle x,\rangle} d x\right) e^{2 \pi i\langle x, m\rangle} \chi_{E} \\
& =P_{E} \sum_{m \in \mathbb{Z}^{d}} \sum_{k \in \mathbb{Z}^{d}}\left\langle P_{\Omega_{k}+k} T_{k} f, E_{m} \chi_{Q}\right\rangle E_{m} \chi_{Q} \\
& =\sum_{k \in \mathbb{Z}^{d}} P_{E} P_{\Omega_{k}+k} T_{k} f=0 .
\end{aligned}
$$

Therefore, for each $f \in L^{2}\left(\mathbb{R}^{d}\right)$ we have

$$
\begin{aligned}
\sum_{m, n \in \mathbb{Z}^{d}}\left\langle f, E_{m} T_{B n} g\right\rangle E_{m} T_{B n} h & =\sum_{m, n \in \mathbb{Z}^{d}}\left\langle T_{-B n} f, E_{m} g\right\rangle T_{B n} E_{m} h \\
& =\sum_{n \in \mathbb{Z}^{d}} T_{B n}\left(\sum_{m \in \mathbb{Z}^{d}}\left\langle T_{-B n} f, E_{m} g\right\rangle E_{m} h\right) \\
& =0 .
\end{aligned}
$$


Hence $\mathbf{G}\left(g, \mathbb{Z}^{d}, \mathcal{K}\right)$ and $\mathbf{G}\left(h, \mathbb{Z}^{d}, \mathcal{K}\right)$ are two orthogonal frames as we claimed, and so the frame representation $\pi$ associated with the lattice $\mathcal{L} \times \mathcal{K}$ has frame multiplicity at least 2 .

Combining the above proof with Corollary 5.4 in the appendix section we have the following more general statement:

Corollary 4.6. Let $\mathcal{L}=A \mathbb{Z}^{d}, \mathcal{K}=B \mathbb{Z}^{d}$ be two full rank lattices in $\mathbb{R}^{d}$. Then the following are equivalent:

(i) The associated Gabor projective unitary representation $\pi$ of $\mathcal{L} \times \mathcal{K}$ has frame multiplicity $N$,

(ii) $\frac{1}{N+1}<|\operatorname{det}(A B)| \leq \frac{1}{N}$.

\section{Appendix: Lattice tiling}

The main purpose of this appendix is to prove Theorem 4.4. The proof follows the same line as in the proof of Theorem 1.2 in [25], except for taking care of the volume of $\mathcal{K}$. We include the sketch of the proof for completeness. Without losing generality, we will assume that $\mathcal{L}=\mathbb{Z}^{d}$ and $\mathcal{K}=A \mathbb{Z}^{d}$ with $|\operatorname{det} A| \leq \frac{1}{2}$. We use $M_{d}(\mathbb{Z})\left(\right.$ resp. $\left.M_{d}(\mathbb{Q})\right)$ to denote the set of all $d \times d$ matrices with entries in $\mathbb{Z}$ (resp. in $\mathbb{Q})$.

Lemma 5.1. Let $P, Q \in M_{d}(\mathbb{Z})$ be unimodular matrices (i.e. $|\operatorname{det} P|=|\operatorname{det} Q|=$ 1). Then Theorem 4.4 holds for $A$ if and only if it holds for PAQ.

Proof. Suppose that there exist $\Omega$ and $E$ such that both $\Omega$ and $E$ tile $\mathbb{R}^{d}$ by $\mathcal{K}$ and pack $\mathbb{R}^{d}$ by $\mathcal{L}$, and $(\Omega+\ell) \cap\left(E+\ell^{\prime}\right)$ has Lebesgue measure 0 for any $\ell, \ell^{\prime}$ in $\mathcal{L}$. Then

$$
\begin{aligned}
P(\Omega)+P A \mathbb{Z}^{d} & =P(\Omega)+P A Q Q^{-1} \mathbb{Z}^{d} \\
& =P(\Omega)+P A Q \mathbb{Z}^{d}
\end{aligned}
$$

and

$$
P(\Omega)+P \mathbb{Z}^{d}=P(\Omega)+\mathbb{Z}^{d} .
$$

Thus $P(\Omega)$ tiles by $\mathcal{K}$ and packs by $\mathcal{L}$. Moreover

$$
(P(\Omega)+\ell) \cap\left(P(E)+\ell^{\prime}\right)=P\left[\left(\Omega+P^{-1} \ell_{1}\right) \cap\left(E+P^{-1} \ell_{2}\right)\right]
$$

has Lebesgue measure 0 for any $\ell, \ell^{\prime}$ in $\mathcal{L}$. Therefore Theorem 20 holds for $P A Q$. Conversely, if Theorem 4.4 holds for $P A Q$, then it follows immediately that it also holds for $A=P^{-1}(P A Q) Q^{-1}$ since $P^{-1}, Q^{-1}$ are unimodular matrices in $M_{d}(\mathbb{Z})$.

The torus $\mathbb{R}^{d} / \mathbb{Z}^{d}$ is denoted by $\mathbb{T}^{d}$, and $\pi_{d}: \mathbb{R}^{d} \rightarrow \mathbb{T}^{d}$ denotes the canonical map. The Haar measure of $\mathbb{T}^{d}$ will be denoted by $\nu(\cdot)$, with $\nu\left(\mathbb{T}^{d}\right)=1$. A subset $S \subseteq \mathbb{T}^{d}$ is called a subspace if $S=\pi_{d}(V)$ where $V \subset \mathbb{R}^{d}$ is a linear subspace. The subspace $S$ is called rational if $V$ is rational, i.e. it has a basis consisting of vectors in $\mathbb{Q}^{d}$.

Lemma 5.2 ([25]). Let $G$ be a closed subgroup of $\mathbb{T}^{d}$. Then

$$
G=S \oplus F
$$

where $S$ is a rational subspace of $\mathbb{T}^{d}$ and $F$ is a finite group. 
For any $s \in \mathbb{T}^{d}$ let $\tau_{s}$ denote the translation $\tau_{s}(x)=x+s$ in $\mathbb{T}^{d}$. Suppose that $\Omega \subseteq \mathbb{T}^{d}$ and $S$ is a countable subset of $\mathbb{T}^{d}$. We say that $\tilde{\Omega}$ is $S$-shifted from $\Omega$, or $\tilde{\Omega}$ is an $S$-shift of $\Omega$, if $\Omega$ has a measure disjoint partition $\Omega=\bigcup_{s \in S} \Omega^{(s)}$ such that

$$
\tilde{\Omega}=\bigcup_{s \in S} \tau_{s}\left(\Omega^{(s)}\right),
$$

where the above union is measure disjoint. We say a subset $\Omega$ of $\mathbb{T}^{d}$ is a polytope (respectively, cube, parallelepiped, etc.) if it is the projection of a polytope (respectively, cube, parallelepiped, etc.) in $\mathbb{R}^{d}$. Similarly, for any $s \in \mathbb{R}^{d}$ we denote $\tau_{s}(x):=x+s$ (a slight abuse of notation). Let $S$ be a countable subset of $\mathbb{R}^{d}$ and $\Omega \subseteq \mathbb{R}^{d}$. We say that $\tilde{\Omega}$ is an $S$-shift of $\Omega$ if $\Omega$ has a measure disjoint partition $\Omega=\bigcup_{s \in S} \Omega^{(s)}$ such that

$$
\tilde{\Omega}=\bigcup_{s \in S} \tau_{s}\left(\Omega^{(s)}\right),
$$

where the above union is measure disjoint.

Lemma 5.3. Let $S$ be a countable subset of $\mathbb{R}^{d}$ such that $\pi_{d}(S)$ is dense in $\mathbb{T}^{d}$. Let $\Omega$ and $R$ be finite unions of polytopes in $\mathbb{R}^{d}$ and $\mathbb{T}^{d}$, respectively, with $\mu(\Omega) \leq \nu(R)$. Then there exists an $S$-shift $\tilde{\Omega}$ of $\Omega$ such that $\pi_{d}: \tilde{\Omega} \longrightarrow R$ is one-to-one.

Let $\mathcal{L}$ be any full-rank lattice in $\mathbb{R}^{d}$. Two measurable sets $\Omega_{1}$ and $\Omega_{2}$ are said to be $\mathcal{L}$-congruent if $\Omega_{1}$ is an $\mathcal{L}$-shift of $\Omega_{2}$. Clearly, if $\Omega_{1}$ and $\Omega_{2}$ are $\mathcal{L}$-congruent, then $\Omega_{1}$ tiles (packs) by $\mathcal{L}$ if and only if $\Omega_{2}$ does.

Proof of Theorem 4.4. Without loss of generality we may assume that $\mathcal{L}=\mathbb{Z}^{d}$ and $\mathcal{K}=A \mathbb{Z}^{d}$ where $A \in M_{d}(\mathbb{R})$ with $|\operatorname{det} A| \leq \frac{1}{2}$. Since $\overline{\pi_{d}(\mathcal{K})}$ is a closed subgroup of $\mathbb{T}^{d}$, by Lemma 5.2 we have $\overline{\pi_{d}(\mathcal{K})}=S \oplus F$ for some rational subspace $S$ and finite set $F$.

Case I. $S=\mathbb{T}^{d}$

Under this condition $\pi_{d}(\mathcal{K})=\pi_{d}\left(A \mathbb{Z}^{d}\right)$ is dense in $\mathbb{T}^{d}$. Let $\Omega_{1}$ be the parallelepiped spanned by the columns of $A$. Then $\Omega_{1}$ tiles $\mathbb{R}^{d}$ by $\mathcal{K}$ and $\mu\left(\Omega_{1}\right)=$ $|\operatorname{det} A| \leq \frac{1}{2}$. Let $R_{1}$ and $R_{2}$ be two disjoint subsets of $\mathbb{T}^{d}$ such that they both are finite unions of polytopes and $\nu\left(R_{1}\right)=\nu\left(R_{2}\right)=\frac{1}{2}$. Then, by Lemma 5.3 , there exists a $\mathcal{K}$-shift $\Omega$ of $\Omega_{1}$ such that $\pi: \Omega \longrightarrow R_{1}$ is one-to-one. Hence $\Omega$ packs by $\mathbb{Z}^{d}$. Since $\Omega$ is $\mathcal{K}$-congruent to $\Omega_{1}$, so it tiles by $\mathcal{K}$. By the same reason, there exists another $\mathcal{K}$-shift $E$ of $\Omega_{1}$ such that $\pi: E \longrightarrow R_{2}$ is one-to-one, and therefore $E$ packs by $\mathbb{Z}^{d}$ and tiles by $\mathcal{K}$. Since $R_{1}$ and $R_{2}$ are disjoint, we also have that $(\Omega+\ell)$ and $\left(E+\ell^{\prime}\right)$ are disjoint for any $\ell, \ell^{\prime}$ in $\mathcal{L}$.

Case II. $S=\{0\}$

In this case $A \in M_{d}(\mathbf{Q})$ is a rational matrix. Write $A=\frac{1}{q} \tilde{A}$ with $\tilde{A} \in M_{d}(\mathbb{Z})$ for some $q \in \mathbb{Z}$. The Smith canonical form (see Newman [30]) for $\tilde{A}$ implies that there exist unimodular integral matrices $P, Q$ such that

$$
P \tilde{A} Q=\left[\begin{array}{cccc}
r_{1} & 0 & \cdots & 0 \\
0 & r_{2} & \cdots & 0 \\
\vdots & \vdots & \ddots & \vdots \\
0 & 0 & \cdots & r_{d}
\end{array}\right]
$$


where each $r_{i} \in \mathbb{Z}$ and $r_{i} \mid r_{i+1}$. By Lemma 5.1, we may assume that

$$
A=\frac{1}{q} \operatorname{diag}\left(r_{1}, r_{2}, \ldots, r_{d}\right),
$$

where each $r_{i} \in \mathbb{Z}$ and $r_{i} \mid r_{i+1}$.

Write

$$
A=\operatorname{diag}\left(\frac{p_{1}}{q_{1}}, \frac{p_{2}}{q_{2}}, \ldots, \frac{p_{d}}{q_{d}}\right), \text { with }\left(p_{i}, q_{i}\right)=1 .
$$

The rectangular parallelepiped spanned by the columns of $A$ is

$$
\Omega_{1}=\left[0, \frac{p_{1}}{q_{1}}\right) \times \cdots \times\left[0, \frac{p_{d}}{q_{d}}\right),
$$

which is a fundamental domain of $\mathcal{K}$. Let $T$ be the smaller rectangular parallelepiped $T=\left[0, \frac{1}{q_{1}}\right) \times \cdots \times\left[0, \frac{1}{q_{d}}\right)$. Then

$$
\Omega_{1}=T+\left\{\left[\frac{k_{1}}{q_{1}}, \ldots, \frac{k_{d}}{q_{d}}\right]^{T}: 0 \leq k_{i}<p_{i}\right\}:=T+\mathcal{F} .
$$

Write the cube $[0,1)^{d}$ in the form of

$$
[0,1)^{d}=T+\left\{\left[\frac{k_{1}}{q_{1}}, \ldots, \frac{k_{d}}{q_{d}}\right]^{T}: 0 \leq k_{i}<q_{i}\right\}:=T+\mathcal{G}
$$

and write

$$
\mathcal{F}=\left\{\alpha_{1}, \alpha_{2}, \ldots, \alpha_{M}\right\}, \mathcal{G}=\left\{\beta_{1}, \beta_{2}, \ldots, \beta_{N}\right\} .
$$

It follows from $|\operatorname{det}(A)| \leq \frac{1}{2}$ that $2 M \leq N$. Then we can prove that there exist $\gamma_{i}, \delta_{i} \in \mathcal{K}$ for each $1 \leq i \leq M$ such that

$$
\alpha_{i}+\gamma_{i} \equiv \beta_{i}(\bmod 1)
$$

and

$$
\alpha_{i}+\delta_{i} \equiv \beta_{M+i}(\bmod 1) .
$$

Now, let $\Omega=T+\left\{\alpha_{i}+\gamma_{i}: 1 \leq i \leq M\right\}$ and $E=T+\left\{\alpha_{i}+\delta_{i}: 1 \leq i \leq\right.$ $M\}$. Since $\gamma_{i}, \delta_{i} \in \mathcal{K}$, we have that both $\Omega$ and $E$ are $\mathcal{K}$-congruent to $\Omega_{1}$ and so both tile $\mathbb{R}^{d}$ by $\mathcal{K}$. It also follows from (5.6) and (5.7) that $\Omega$ is $\mathbb{Z}^{d}$-congruent to $T+\left\{\beta_{i}: 1 \leq i \leq M\right\}$ and $E$ is $\mathbb{Z}^{d}$-congruent to $T+\left\{\beta_{i}: M+1 \leq i \leq 2 M\right\}$. Since both $T+\left\{\beta_{i}: 1 \leq i \leq M\right\}$ and $T+\left\{\beta_{i}: M+1 \leq i \leq 2 M\right\}$ are disjoint subsets of $[0,1)^{d}$, we have that both $\Omega$ and $E$ pack by $\mathbb{Z}^{d}$, and $(\Omega+\ell)$ and $\left(E+\ell^{\prime}\right)$ are disjoint for any $\ell, \ell^{\prime}$ in $\mathcal{L}$.

Case III. None of the above

Here we have $\overline{\mathcal{K}(\bmod 1)}=S \oplus F$ where $S$ is a rational subspace of dimension $e$ with $0<e<d$.

By Sub-Lemma 2 in [25], we may assume that

$$
A=\left[\begin{array}{cc}
A_{1} & B \\
0 & D
\end{array}\right]
$$

with $D=\operatorname{diag}\left(\frac{p_{1}}{q_{1}}, \ldots, \frac{p_{e-d}}{q_{e-d}}\right)$ for $\left(p_{i}, q_{i}\right)=1, q_{i}>0$, and $\left[A_{1} B\right] \mathbb{Z}^{d}(\bmod 1)$ dense in $[0,1]^{e}$. For simplicity denote $r=d-e$. An element $\alpha$ of $\mathcal{K}$ has the form $\alpha=\left[\alpha_{e}, \alpha_{r}\right]^{T}$, in which $\alpha_{e} \in\left[A_{1} B\right] \mathbb{Z}^{d}$ and $\alpha_{r}=\left[\frac{p_{1} m_{1}}{q_{1}}, \ldots, \frac{p_{r} m_{r}}{q_{r}}\right]^{T} \in D \mathbb{Z}^{r}$ for $m_{1}, \ldots, m_{r} \in \mathbb{Z}$.

Let

$$
\tilde{\Omega}=\Omega^{e} \times \Omega^{r}, \text { where } \Omega^{e}=A_{1}\left([0,1)^{e}\right), \Omega^{r}=D\left([0,1)^{r}\right) .
$$


Then $\Omega$ is a fundamental domain of $\mathcal{K}=A \mathbb{Z}^{d}$. Set $T^{r}=\left[0, \frac{1}{q_{1}}\right) \times \cdots \times\left[0, \frac{1}{q_{r}}\right)$. Then

$$
\Omega^{r}=T^{r} \oplus \mathcal{F}_{r}, \text { where } \mathcal{F}_{r}=\left\{\left[\frac{k_{1}}{q_{1}}, \ldots, \frac{k_{r}}{q_{r}}\right]^{T}: 0 \leq k_{i}<p_{i}\right\},
$$

which yields

$$
\tilde{\Omega}=\Omega^{e} \times\left(T^{r} \oplus \mathcal{F}_{r}\right)=\bigcup_{\alpha \in \mathcal{F}_{r}} \Omega^{e} \times\left(T^{r}+\alpha\right) .
$$

Meanwhile, the $\mathbb{Z}^{d}$-tile $[0,1)^{d}$ has a decomposition

$$
[0,1)^{d}=[0,1)^{e} \times[0,1)^{r}=\left(\bigcup_{\alpha \in \mathcal{F}_{r}} R_{\alpha} \times[0,1)^{r}\right) \cup\left(\bigcup_{\alpha \in \mathcal{F}_{r}} \tilde{R}_{\alpha} \times[0,1)^{r}\right),
$$

where $[0,1)^{e}$ is partitioned into $2\left|\mathcal{F}_{r}\right|$ disjoint rectangular parallelepipeds $R_{\alpha}$ and $\tilde{R}_{\alpha}$ of equal volume in $\mathbb{R}^{e}$ indexed by the elements of $\mathcal{F}_{r}$. Since $\mu(\tilde{\Omega}) \leq \frac{1}{2}$ we have

$$
\begin{aligned}
\left|\mathcal{F}_{r}\right| \cdot \mu\left(\Omega^{e} \times\left(T^{r}+\alpha\right)\right) & =\mu(\tilde{\Omega}) \leq \frac{1}{2}=\frac{1}{2} \mu\left([0,1)^{e}\right) \\
& =\frac{1}{2} \cdot 2\left|\mathcal{F}_{r}\right| \cdot \mu\left(R_{\alpha} \times[0,1)^{r}\right) \\
& =\left|\mathcal{F}_{r}\right| \cdot \mu\left(R_{\alpha} \times[0,1)^{r}\right) \\
& =\left|\mathcal{F}_{r}\right| \cdot \mu\left(\tilde{R}_{\alpha} \times[0,1)^{r}\right) .
\end{aligned}
$$

Thus

$$
\mu\left(\Omega^{e} \times\left(T^{r}+\alpha\right)\right) \leq \mu\left(R_{\alpha} \times[0,1)^{r}\right)
$$

and

$$
\mu\left(\Omega^{e} \times\left(T^{r}+\alpha\right)\right) \leq \mu\left(\tilde{R}_{\alpha} \times[0,1)^{r}\right) .
$$

By Sub-Lemma 5 in [25], for each $\alpha \in \mathcal{F}_{r}$ there exist two $\mathcal{K}$-shifts $\Omega_{\alpha}$ and $E_{\alpha}$ of $\Omega^{e} \times\left(T^{r}+\alpha\right)$ such that both $\pi_{d}: \Omega_{\alpha} \longrightarrow R_{\alpha} \times[0,1)^{r}$ and $\pi_{d}: E_{\alpha} \longrightarrow \tilde{R}_{\alpha} \times[0,1)^{r}$ are one-to-one, where we view $R_{\alpha} \times[0,1)^{r}$ and $\tilde{R}_{\alpha} \times[0,1)^{r}$ as subsets of $\mathbb{T}^{d}$.

Now let

$$
\Omega=\bigcup_{\alpha \in \mathcal{F}_{r}} \Omega_{\alpha}
$$

and

$$
E=\bigcup_{\alpha \in \mathcal{F}_{r}} E_{\alpha}
$$

Then both $\Omega$ and $E$ are $\mathcal{K}$-shifts of the fundamental domain $\tilde{\Omega}$ of $\mathcal{K}$. Hence they both tile $\mathbb{R}^{d}$ by $\mathcal{K}$. Since

$$
\pi_{d}: \Omega \longrightarrow \bigcup_{\alpha \in \mathcal{F}_{r}} R_{\alpha} \times\left(T^{r}+\beta\right)=[0,1)^{d} \text { is one-to-one }
$$

and

$$
\pi_{d}: E \longrightarrow \bigcup_{\alpha \in \mathcal{F}_{r}} \tilde{R}_{\alpha} \times\left(T^{r}+\beta\right)=[0,1)^{d} \text { is one-to-one, }
$$

we have that $\Omega$ packs by $\mathbb{Z}^{d}$ and so does $E$. Finally, since the images of $\pi_{d}(\Omega)$ and $\pi_{d}(E)$ are disjoint, we obtain that $(\Omega+\ell)$ and $\left(E+\ell^{\prime}\right)$ are disjoint for any $\ell, \ell^{\prime}$ in $\mathcal{L}$.

Combining all three cases we complete the proof of Theorem 4.4. 
It is not hard to check that the above proof can be easily modified to get the following more general result:

Corollary 5.4. Let $\mathcal{L}, \mathcal{K}$ be two full rank lattices in $\mathbb{R}^{d}$ such that $\mathrm{v}(\mathcal{L}) \geq N \mathrm{v}(\mathcal{K})$. Then there exist $N$ measurable subsets $\Omega_{i}(i=1, \ldots, N)$ of $\mathbb{R}^{d}$ such that

(i) each $\Omega_{i}$ tiles $\mathbb{R}^{d}$ by $\mathcal{K}$ and packs $\mathbb{R}^{d}$ by $\mathcal{L}$;

(ii) $\left(\Omega_{i}+\ell\right) \cap\left(\Omega_{j}+\ell^{\prime}\right)$ has Lebesgue measure 0 for any $\ell, \ell^{\prime}$ in $\mathcal{L}$ and all $i \neq j$.

\section{REFERENCES}

[1] A. Aldroubi, D. Larson, Wai-Shing Tang and E. Weber, Geometric aspects of frame representations of abelian groups, Trans. Amer. Math. Soc., 356 (2004), no. 12, 4767-4786. MR2084397 (2005g:42067)

[2] J. Antezana, G. Corach, M. Ruiz and D. Stojanofff, Oblique projections and frames, Proc. Amer. Math. Soc., 134 (2006), 1031-1037. MR2196035 (2006j:42046)

[3] R. Balan, Equivalence relations and distances between Hilbert frames, Proc. Amer. Math. Soc., 127 (1999), 2353-2366. MR1600096 (99j:46025)

[4] R. Balan, A study of Weyl-Heisenberg and wavelet frames, Ph.D. Thesis, Princeton University, 1998.

[5] R. Balan, P. Casazza, C. Heil and Z. Landau, Deficits and excess of frames, Adv. Comput. Math., 18 (2003), 93-116. MR1968114 (2004a:42040)

[6] P. Casazza, Modern tools for Weyl-Heisenberg (Gabor) frame theory, Adv. Imag. Elect. Phys., 115 (2001), 1-127.

[7] P. Casazza, D. Han and D. Larson, Frames in Banach spaces, Contemp. Math., 247 (1999), 149-182. MR1738089 (2000m:46015)

[8] P. Casazza and J. Tremain, The Kadison-Singer problem in mathematics and engineering, Proc. Natl. Acad. Sci. USA, 103 (2006), 2032-2039. MR2204073 (2006j:46074)

[9] R. J. Duffin and A. C. Schaeffer, A class of nonharmonic Fourier series, Trans. Amer. Math. Soc., 72 (1952), 341-366. MR0047179 (13:839a)

[10] D. Dutkay, The local trace functions for super-wavelets, Contemp. Math., 345 (2004), 115136. MR2066824 (2005g:42077)

[11] D. Dutkay, S. Bildea and G. Picioroaga, MRA Superwavelets, New York Journal of Mathematics, 11 (2005), 1-19. MR2154344 (2006b:42048)

[12] H. G. Feichtinger and T. Strohmer (eds.), Gabor Analysis and Algorithms: Theory and Applications, Applied and Numerical Harmonic Analysis, Birkhäuser, 1998. MR1601119 (98h:42001)

[13] H. G. Feichtinger and T. Strohmer (eds.), Advances in Gabor Analysis, Applied and Numerical Harmonic Analysis, Birkhäuser, 2002.

[14] M. Frank and D. Larson, Frames in Hilbert $C^{*}$-modules and $C^{*}$-algebras, J. Operator Theory, 48 (2002), 273-314. MR1938798 (2003i:42040)

[15] M. Frank, V. Paulsen and T. Tiballi, Symmetric approximation of frames and bases in Hilbert spaces, Trans. Amer. Math. Soc., 354 (2002), 777-793. MR1862567 (2002j:42042)

[16] J-P. Gabardo and D. Han, Frame representations for group-like unitary operator systems, $J$. Operator Theory, 49 (2003), 223-244. MR1991737 (2004e:42047)

[17] J-P. Gabardo and D. Han, Aspects of Gabor analysis and operator algebras. Advances in Gabor analysis, 129-152, Appl. Numer. Harmon. Anal., Birkhäuser Boston, Boston, MA, 2003. MR1955934

[18] J-P. Gabardo and D. Han, The uniqueness of the dual of Weyl-Heisenberg subspace frames, Appl. Comput. Harmon. Anal., 17 (2004), 226-240. MR2082160 (2005g:43012)

[19] D. Gabor, Theory of Communication, J. Inst. Elec. Eng. (London) 93 (1946), 429-457.

[20] K. Gröchenig, Foundations of Time-Frequency Analysis, Applied and Numerical Harmonic Analysis, Birkhäuser, 2001. MR1843717 (2002h:42001)

[21] K. Gröchenig and M. Leinert, Wiener's lemma for twisted convolution and Gabor frames, $J$. Amer. Math. Soc., 17 (2004), 1-18. MR2015328 (2004m:42037)

[22] D. Han, Approximations for Gabor and wavelet frames, Trans. Amer. Math. Soc., 355 (2003), 3329-3342. MR1974690 (2004j:42027)

[23] D. Han and D. Larson, Frames, bases and group parametrizations, Memoirs Amer. Math. Soc., 697 (2000). 
[24] D. Han and D. Larson, Wandering vector multipliers for unitary groups, Trans. Amer. Math. Soc., 353 (2001), 3347-3370. MR1828609 (2002c:46116)

[25] D. Han and Y. Wang, Lattice tiling and Weyl-Heisenberg frames, Geometric and Functional Analysis, 11 (2001), 742-758. MR1866800 (2003j:52021)

[26] D. Han and Y. Wang, The existence of Gabor bases, Contemp. Math., 345 (2004), 183-192. MR2066828 (2005f:42069)

[27] C. Heil, P. Jorgensen and D. Larson, Wavelets, frames and operator theory, Contemp. Math., 345 (2004). MR2066817 (2004m:42001)

[28] R. Kadison and J. Ringrose, Fundamentals of the Theory of Operator Algebras, Vols. I and II, Academic Press, Inc., 1983 and 1985. MR719020 (85j:46099)

[29] D. Larson, Frames and wavelets from an operator-theoretic point of view, Contemp. Math., 228 (1998), 201-218. MR1667663 (2000e:47112)

[30] M. Newman, Integral Matrices, Academic Press, New York, 1972. MR0340283 (49:5038)

[31] V. S. Varadarajan, Geometry of Quantum Theory, Second Edition, Springer-Verlag, New York-Berlin, 1985. MR805158 (87a:81009)

[32] Eric Weber, Orthogonal frames of translates, Appl. Comput. Harmon. Anal., 17 (2004), 69-90. MR2067916 (2005h:42064)

Department of Mathematics, University of Central Florida, Orlando, Florida 32816

E-mail address: dhan@pegasus.cc.ucf.edu 